\title{
Ketamine promotes the neural differentiation of mouse embryonic stem cells by activating mTOR
}

\author{
XUHUI ZHOU* ${ }^{*}$ XIANG LV*, LEI ZHANG, JIA YAN, RONG HU, YU SUN, SIWEI XI and HONG JIANG \\ Department of Anesthesiology, Shanghai Ninth People's Hospital, \\ Shanghai Jiao Tong University School of Medicine, Center for Specialty Strategy Research of \\ Shanghai Jiao Tong University China Hospital Development Institute, Shanghai 200011, P.R. China
}

Received December 27, 2017; Accepted August 31, 2018

DOI: $10.3892 / \mathrm{mmr} .2020 .11043$

\begin{abstract}
Ketamine is a widely used general anesthetic and has been reported to demonstrate neurotoxicity and neuroprotection. Investigation into the regulatory mechanism of ketamine on influencing neural development is of importance for a better and safer way of relieving pain. Reverse transcription-quantitative polymerase chain reaction and western blotting were used to detect the critical neural associated gene expression, and flow cytometry to detect the neural differentiation effect. Hence, in the present study the underlying mechanism of ketamine $(50 \mathrm{nM})$ on neural differentiation of the mouse embryonic stem cell (mESC) line 46C was investigated. The results demonstrated that a low dose of ketamine $(50 \mathrm{nM})$ promoted the differentiation of mESCs to neural stem cells (NSCs) and activated mammalian target of rapamycin (mTOR) by upregulating the expression levels of phosphorylated (p)-mTOR. Furthermore, inhibition of the mTOR signaling pathway by rapamycin or knockdown of mTOR suppressed neural differentiation. A rescue experiment further confirmed that downregulation of mTOR inhibited the promotion of neural differentiation induced by ketamine. Taken together, the present study indicated that a low level of ketamine upregulated p-mTOR expression levels, promoting neural differentiation.
\end{abstract}

\section{Introduction}

Ketamine, an N-methyl-D-aspartate (NMDA) receptor antagonist, is widely used in pediatric anesthesia, periop-

Correspondence to: Professor Hong Jiang, Department of Anesthesiology, Shanghai Ninth People's Hospital, Shanghai Jiao Tong University School of Medicine, Center for Specialty Strategy Research of Shanghai Jiao Tong University China Hospital Development Institute, 639 Zhizaoju Road, Shanghai 200011, P.R. China

E-mail: jianghongjiuyuan@163.com

*Contributed equally

Key words: ketamine, mammalian target of rapamycin, neural differentiation, mouse embryonic stem cells erative sedation, analgesia and other diagnostic procedures in pediatrics for children 0-14 years old (1). It is often consumed as a drug of abuse by the public, including pregnant women (2); the fetuses of such pregnant patients, who received non-obstetric surgery, have an increasing incidence of exposure to ketamine through the placenta. Additionally, $0.75-2 \%$ of pregnant women require surgery associated with pregnancy or other medical issues $(3,4)$. A series of experiments have revealed that ketamine can induce neuroapoptosis and damage in the developing brain (5-7). Repeated exposure to ketamine can be deleterious to neurodevelopment in infants (8). In contrast, increasing evidence also suggested that ketamine has neuroprotective function. Clinical studies have demonstrated that a single dose of ketamine mitigates postoperative cognitive dysfunction (8) and may offer specific protection towards post-operative cognitive dysfunction (9). Ketamine may additionally prevent stress-induced cognitive inflexibility in rats (10). Previous studies demonstrated that for traumatic brain injuries (TBIs), subarachnoid hemorrhage, malignant stroke and other neurological diseases, ketamine could inhibit the neuronal discharge across all injury modalities $(11,12)$. The neuroprotective function of ketamine has also been demonstrated in hypoxia-ischemia and TBI, and as a fast-acting antidepressant (13-15). Dong et al (16) demonstrated that the phosphoinositide 3-kinase-protein kinase B/Akt signaling pathway was involved in ketamine-induced neurogenesis of cultured neural stem/progenitor cells (NSPCs). Furthermore, ketamine induces human neurotoxicity in neurons differentiated from human embryonic stem cells (hESCs) via the reactive oxygen species-mediated mitochondrial apoptosis pathway (17). These studies suggested that the effect of ketamine on neurodevelopment may be dose-dependent. Additionally, the underlying mechanism of ketamine on neurodevelopment may also depend on different developmental stages; however, the molecular mechanism of ketamine regulating the early development of neural cells remains unclear.

Mouse ESCs (mESCs) derived from embryos at the pre-implantation stage demonstrating an unlimited self-renewal ability and capacity to generate different cell types are valuable for clinical research (18). Therefore, mESCs are an important as an in vitro model to study ontogenetic development. Previous studies identified that there are specific 
critical genes regulating neural differentiation, for example, zing finger homeobox (Zfhx)1b has been reported to promote neural stem cell (NSC) colony formation by inducing Sex determining region Y-box (Sox)1 expression (19). Sirtuin1 could mediate alterations in DNA methylation to modulate embryonic stem cell differentiation (20). The microRNA-134/ methyl-CpG binding domain protein 3 axis could regulate the reprogramming and pluripotency of induced pluripotent stem cells, a type of ESC-like cells, from neural progenitor cells (NPCs) (21); however, the neuroprotective function of ketamine in mESCs on NSC differentiation and its downstream mechanism remains elusive.

Mammalian target of rapamycin (mTOR) is a critical regulator of growth and homeostasis (22-24). A growing number of studies have demonstrated that the mTOR-related signaling pathway is associated with the differentiation of NPCs and NSCs $(25,26)$, and is important to regulate oligodendrocyte differentiation and remyelination (27). mTOR also serves an important role in regulating cortical interneuron number and autophagy during brain development (28). Rapamycin, the mechanistic target of mTOR, has been associated with improvements in neurological deficits and increased brain water content (29). However, whether mTOR could regulate the neural differentiation of ESCs has been rarely evaluated. Besides, whether mTOR participates in ketamine regulatory signaling pathway or not, is also unclear.

In the present study, it was determined whether ketamine was able to influence the neural differentiation from the $\mathrm{mESCs}$ and the marker expression of sex-determining region Y-box (Sox)1 (30), N-cadherin (N-cad) (31) and Nestin (32). The present study suggested a safe dose of ketamine for clinical application and demonstrated that mTOR may be a potential target of better and safer therapeutics in the future.

\section{Materials and methods}

mESC culture. The mESC line $46 \mathrm{C}$, containing the Sox 1 promoter and expressing green fluorescence protein (GFP), was employed to indicate the endogenous Sox 1 expression during the neural differentiation at NPCs stage and gifted by Dr Xiaoqing Zhang (Tongji University, Shanghai, China) (33). Cells were cultured on feeder cells that are the irradiated mouse embryonic fibroblasts in KnockOut ${ }^{\mathrm{TM}}$ Dulbecco's modified Eagle's medium (Gibco; Thermo Fisher Scientific, Inc., Waltham, MA, USA; cat. no. 10829018) with $15 \%$ fetal bovine serum (Gibco; Thermo Fisher Scientific, Inc.), leukemia inhibitory factor (Merck KGaA, Darmstadt, Germany; cat. no. LIF2050) and $\beta$-mercaptoethanol ( $\beta$-Me; $1: 10,000$, Sigma-Aldrich; Merck KGaA) at $37^{\circ} \mathrm{C}$, under a $5 \% \mathrm{CO}_{2}$ atmosphere. After $48 \mathrm{~h}$, mESCs were digested into single cells using $0.05 \%$ trypsin (Gibco; Thermo Fisher Scientific, Inc.; cat. no. 2520056) and seeded on new feeder cells for passaging. The feeder cells that were able to secrete leukemia inhibitory factor to support the growth of the ESCs were made in our lab. Feeder cells were made from x-irradiated day 13.5 embryonic fibroblasts. Day 13.5 embryonic fibroblasts were granted from Dr Liu lab in Tongji University.

Neural differentiation of mESCs to NSCs. The protocol was adapted from a previous study (34). The mESC line, 46C, was dissociated into single cells by trypsin and counted. Subsequently, $2 \times 10^{4}$ cells $/ \mathrm{ml} \mathrm{mESCs}$ were washed with Glasgow's minimum essential medium (GMEM; Gibco; Thermo Fisher Scientific, Inc.) in a $6 \mathrm{~cm}$ dish and re-suspended in GMEM with $8 \%$ knockout serum replacement (Gibco; Thermo Fisher Scientific, Inc.), $1 \%$ sodium pyruvate, $1 \%$ L-glutamine (Thermo Fisher Scientific, Inc.), $0.1 \mathrm{mM} \beta$-Me. Cells were cultured in a $6 \mathrm{~cm}$ ultra-low attachment petri dish and passaged every 2 days at $37^{\circ} \mathrm{C}$ in a $5 \% \mathrm{CO}_{2}$ atmosphere. The culture medium was changed every day. Clones exhibiting GFP fluorescence at the stage of NSC derived from $46 \mathrm{C} \mathrm{mESCs}$ were detected with an IX73 + DP80 inverted fluorescence microscope (Olympus Corporation, Tokyo, Japan; magnification, x20). During the neural differentiation, ketamine (final concentration $50 \mathrm{nM}$ ) was added into the medium. The control group consisted of cells treated only with physiological saline $(0.9 \% \mathrm{NaCl})$. For the treatment with MK- $801,10 \mu \mathrm{g} / \mathrm{ml}$ MK-801 was used to treat cells during neural differentiation. Rapamycin (final concentration $50 \mu \mathrm{M}$ ) was added to the medium during the neural differentiation. The control group was treated with dimethyl sulfoxide, which was additionally used as the solvent for rapamycin. All the treating or control culture media was changed every day during the 7 days of neural differentiation from mESCs.

Reverse-transcription quantitative polymerase chain reaction ( $R T-q P C R)$. Total neural stem cell RNA was isolated by RNaiso plus (Takara Biotechnology Co., Ltd., Dalian, China), mRNA was reverse transcribed to cDNA at $37^{\circ} \mathrm{C}$ for 15 min using a RT reagent kit (Perfect Real Time; Takara Biotechnology Co., Ltd.). qPCR was performed using SYBR Green qPCR Mix (Takara Biotechnology Co., Ltd.). The primers are as follows: Nestin forward, 5'-CCCTGAAGT CGAGGAGCTG-3' and reverse, 5'-CTGCTGCACCTCTAA GCGA-3'; N-cadherin forward, 5'AGCGCAGTCTTACCGAA GG-3' and reverse, 5'-TCGCTGCTTTCATACTGAACTTT-3'; Sox 1 forward, 5'-AAGGAACACCCGGATTACAAGT-3' and reverse, 5'-GTTAGCCCAGCCGTTGAC-3'; and GAPDH forward, 5'-AGGTCGGTGTGAACGGATTTG-3' and reverse 5'-TGTAGACCATGTAGTTGAGGTCA-3'. The PCR thermocycling conditions were as follows: Initial denaturation at $95^{\circ} \mathrm{C}$ for $5 \mathrm{~min}$, followed by 40 cycles of denaturation at $95^{\circ} \mathrm{C}$ for $5 \mathrm{sec}$, primer annealing at $60^{\circ} \mathrm{C}$ for $20 \mathrm{sec}$, elongation at $70^{\circ} \mathrm{C}$ for $10 \mathrm{sec}$. In total, three independent experiments were performed. The relative gene expression was presented as $2^{-\Delta \Delta C q}$ using the relative quantification method and normalized to the expression of GAPDH (35).

Western blotting. Cells were lysed by radioimmunoprecipitation assay lysis buffer (Beyotime Institute of Biotechnology, Haimen, China; cat. no. P0013B) and quantified by a Bicinchoninic Protein Assay Kit (Beyotime Institute of Biotechnology; cat. no. P0009). A total of $40 \mu \mathrm{g}$ protein was loaded for electrophoresis on 10\% SDS-PAGE gels. Proteins were transferred onto polyvinylidene fluoride membranes (Merck KGaA; cat. no. MH0323) and blocked with TBS and Tween 20 with 3\% bovine serum albumin (Amresco, Inc., Framingham, MA, USA) for $1 \mathrm{~h}$ at room temperature and incubated with primary antibodies at $4^{\circ} \mathrm{C}$ overnight. The antibodies were as follows: mTOR (cat. no. 2972, Cell Signaling 
A
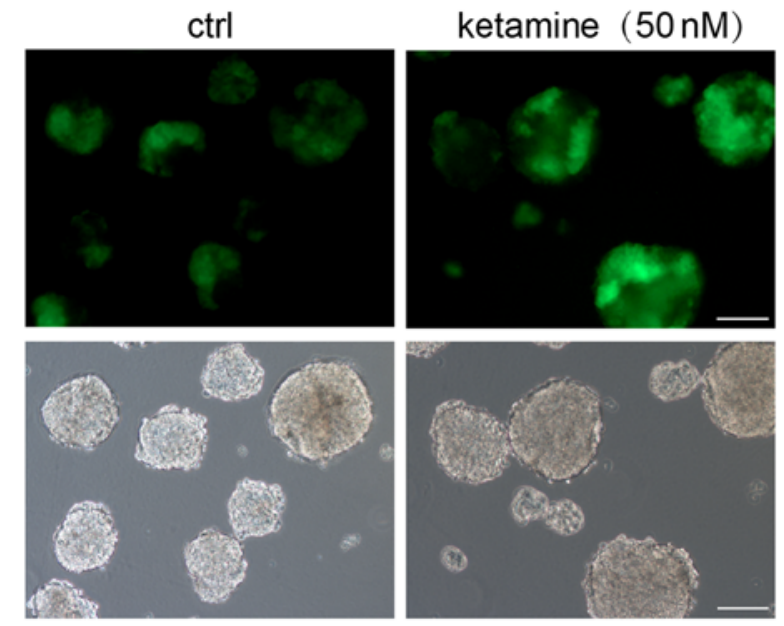

B

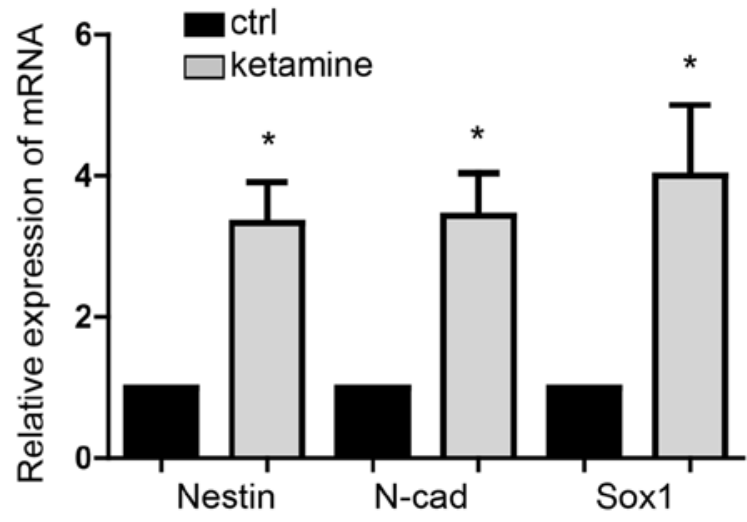

C

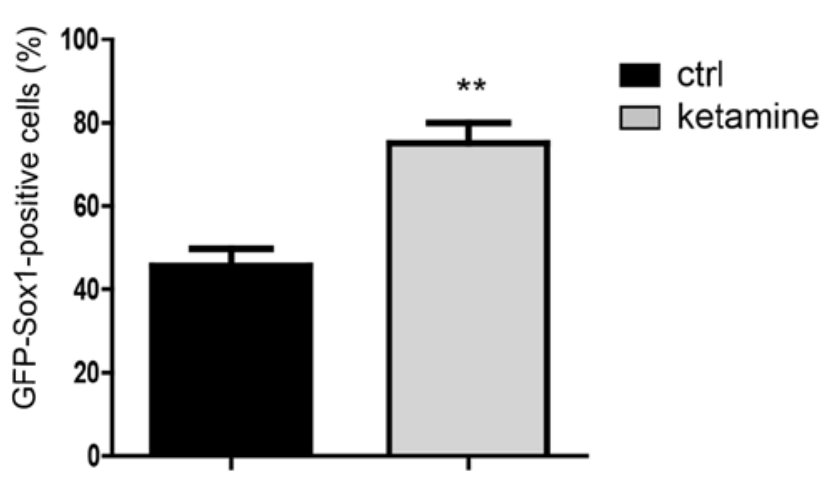

Figure 1. Ketamine promotes neural differentiation. (A) GFP indicated the Sox 1 expression of NPCs, suggesting the differentiation potential. Scale bar, $100 \mu \mathrm{m}$. (B) Detection of the NPCs markers expression by RT-qPCR. (C) Flow cytometry analysis indicated more GFP-Sox1-positive cells in the ketamine-treatment group. Data are presented as the mean \pm standard deviation $(n=3) .{ }^{*} \mathrm{P}<0.05,{ }^{* *} \mathrm{P}<0.01$ vs. the ctrl. Ctrl, control; GFP, green fluorescence protein; NPC, neural progenitor cell; RT-qPCR, reverse transcription-quantitative polymerase chain reaction; Sox, sex-determining region Y-box.

Technology, Inc., Danvers, MA, USA, 1:1,000), p-mTOR (cat. no. 5536, Cell Signaling Technology, Inc., 1:1,000), GAPDH (cat. no. 5174, Cell Signaling Technology, Inc. 1:1,500), p-p70 S6k antibody (cat. no. 9205, Cell Signaling Technology, Inc. 1:1,000) and p70 S6k antibody (cat. no. 2708, Cell Signaling Technology, Inc. 1:1,000). The horseradish peroxidase-conjugated secondary antibody used was anti-rabbit IgG (cat. no. 7074; Cell Signaling Technology, Inc.; 1:2,500) and was incubated with the membranes for $2 \mathrm{~h}$ at room temperature. The bands were detected by an enhanced chemiluminescence western blotting substrate (Thermo Fisher Scientific, Inc.). Amersham Imager 600 (GE Healthcare, Chicago, IL, USA) was used for detecting the signaling. ImageJ_v1.8.0 software (National Institutes of Health, Bethesda, MD, USA) was used for densitometry.

Knockdown of mTOR. The pLKO.1-puro vector (Addgene, Inc, Cambridge, MA, USA; cat. no. 8453) containing mTOR short hairpin (sh)RNA was constructed to downregulate mTOR expression. The sequence of shRNA-1 was: 5'-AGT ACTGTAGCACCTTGGG-3' and of shRNA-2 was: 5'-TCT TCTCTCTGTAGTCCCG-3'. The control vector used was the empty pLKO.1-puro vector. The vectors ( $1 \mu \mathrm{g} / 6 \mathrm{~cm}$ dish) were transiently transfected into the cells during neural differentiation from mESCs at day 3 using the Lipofectamine ${ }^{\circledR} 2000$ Transfection Reagent (Thermo Fisher Scientific, Inc.) and re-transfected at day 5 in order to maintain the knockdown effect during the 7 days of neural transfection. Transfection efficiency was detected by RT-qPCR at day 7 .

Flow cytometry. The mESC line, 46C is a cell line with GFP expression, indicating endogenous Sox 1 expression during the differentiation from mESCs to NSCs. Flow cytometry was performed to detect the quantitative proportion of GFP-Sox1-positive cells to determine the differentiation efficiency. Clones of NSCs were digested to a single cell suspension by $0.25 \%$ trypsin (Gibco; Thermo Fisher Scientific, Inc.; cat. no. 2520056) at $37^{\circ} \mathrm{C}$ for $2 \mathrm{~min}$. Cells were collected by centrifugation at $1,000 \mathrm{xg}$ for $2 \mathrm{~min}$ at room temperature and re-suspended with PBS to wash the cells. This step was repeated twice. The cell suspension in PBS was used for further analysis. A flow cytometer (BD Biosciences, Franklin Lakes, NJ, USA) was used to detect the GFP-Sox1-positive NSCs. The results were analyzed by using FlowJo software (version 7.6.1; FlowJo LLC, Ashland, OR, USA).

Statistical analysis. Each experiment was performed at least 3 times $(n \geq 3)$. Statistical significance was detected by a Student's t-test between two groups. For multiple groups, one-way analysis of variance was used, followed by Tukey's honest significance test. Data are presented as the mean \pm standard deviation. $\mathrm{P}<0.05$ was considered to indicate a statistically significant difference. 
A
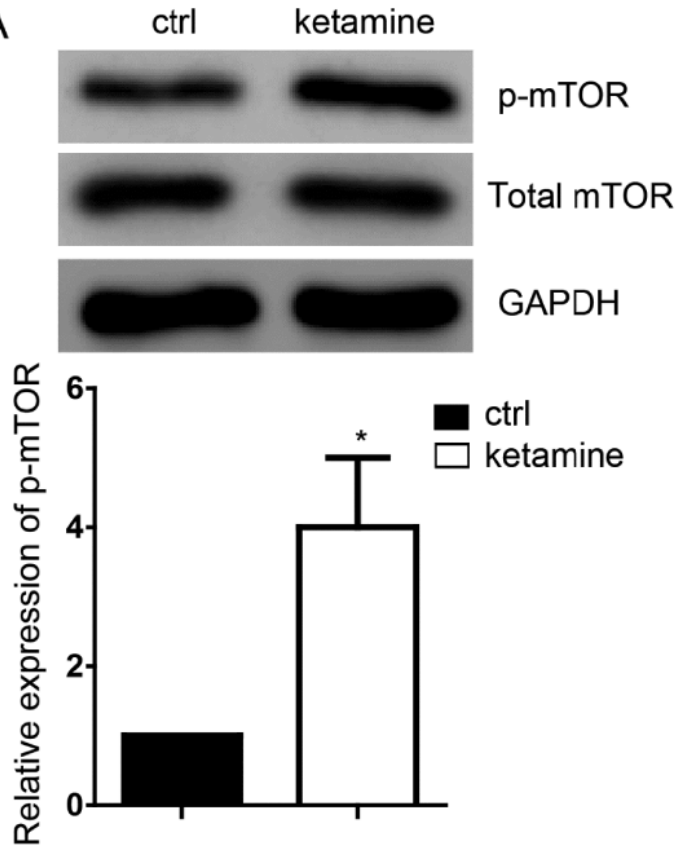

C

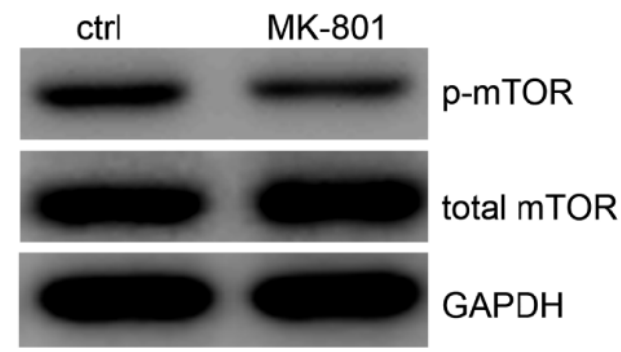

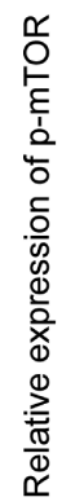

B
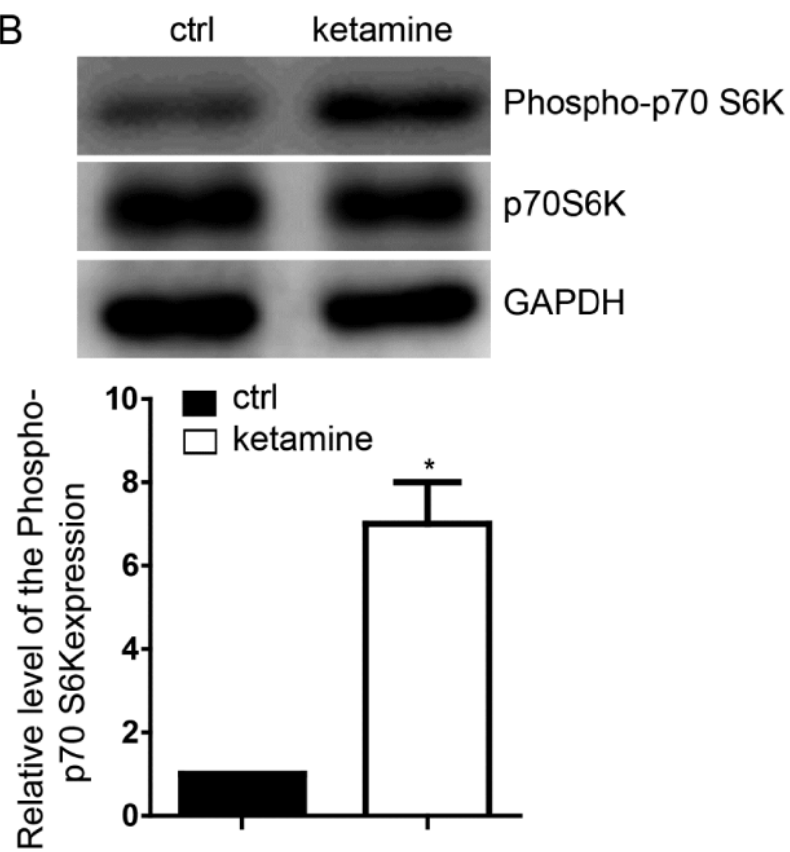

D
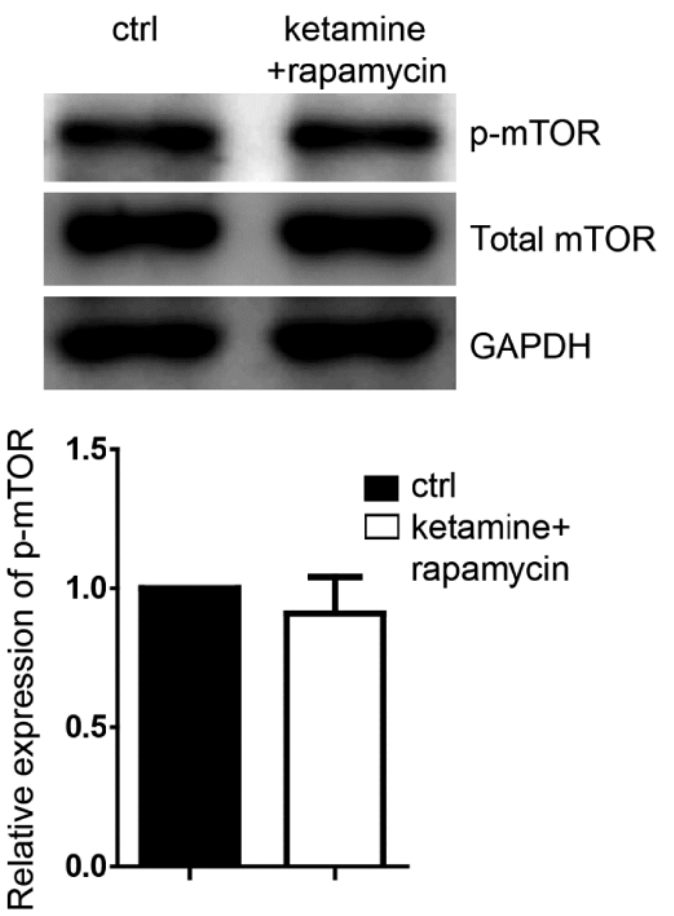

Figure 2. Ketamine activates the mTOR signaling pathway. (A) Representative images of the expression of p-mTOR in neural progenitor cells derived from ESCs as detected by western blotting; the ratio of p-mTOR normalized to GAPDH/total mTOR is presented. (B) Ketamine-treatment group demonstrated the upregulation of p-70SK6. (C) Inhibtion of the NMDA signaling pathway by the NMDA receptor antagonist MK-801 decreased the expression levels of $\mathrm{p}$-mTOR; the ratio of $\mathrm{p}$-mTOR normalized to GAPDH/total mTOR is presented. (D) $50 \mu \mathrm{M}$ rapamycin markedly reduced the activation of the mTOR signaling, which attenuated the function of ketamine on regulating the level of p-mTOR. Data are presented as the mean \pm standard deviation ( $\mathrm{n}=3) .{ }^{*} \mathrm{P}<0.05,{ }^{* *} \mathrm{P}<0.01$ vs. the ctrl. Ctrl, control; ESC, embryonic stem cell; NMDA, N-methyl-D-aspartate; mTOR, mammalian target of rapamycin; p, phosphorylated.

\section{Results}

Ketamine promotes neural differentiation. Neural differentiation of mESCs to NSCs demonstrated that $50 \mathrm{nM}$ ketamine added into the medium significantly promoted the neural differentiation detected on day 7 (Fig. 1A). Subsequently, the expression of NSCs markers was investigated demonstrating that Nestin, N-cad and Sox1 were significantly upregulated in the ketamine-treatment group compared with the control group (Fig. 1B). Flow cytometry further confirmed that the proportion of GFP-Sox1-positive cells was significantly higher in the ketamine-treatment group compared with the control group (Fig. 1C). These results indicated that ketamine may not only be an anesthetic; however, additionally regulates neural differentiation. This suggested the potential influence of ketamine on individual neural differentiation at the early development stage. 
A
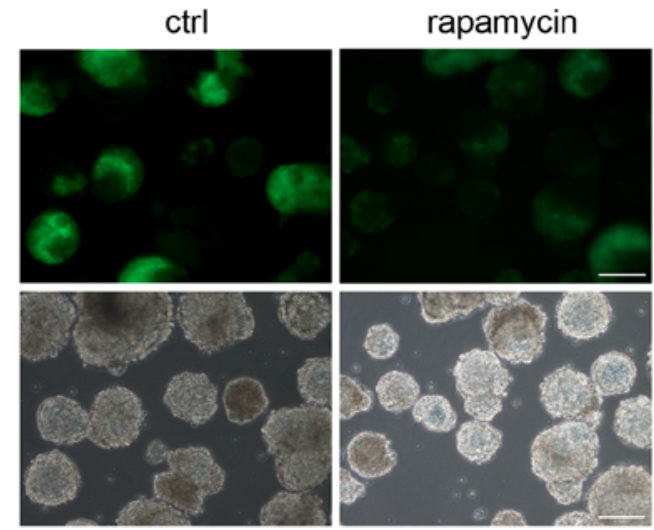

$\mathrm{C}$
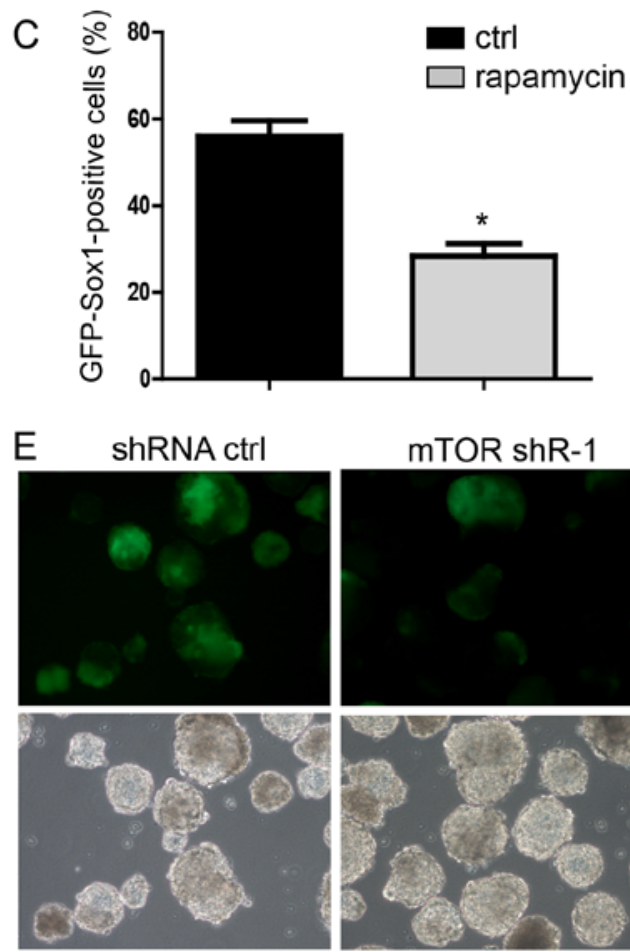

$\mathrm{G}$

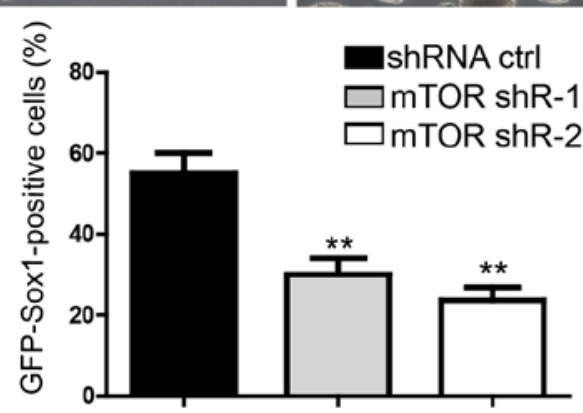

B
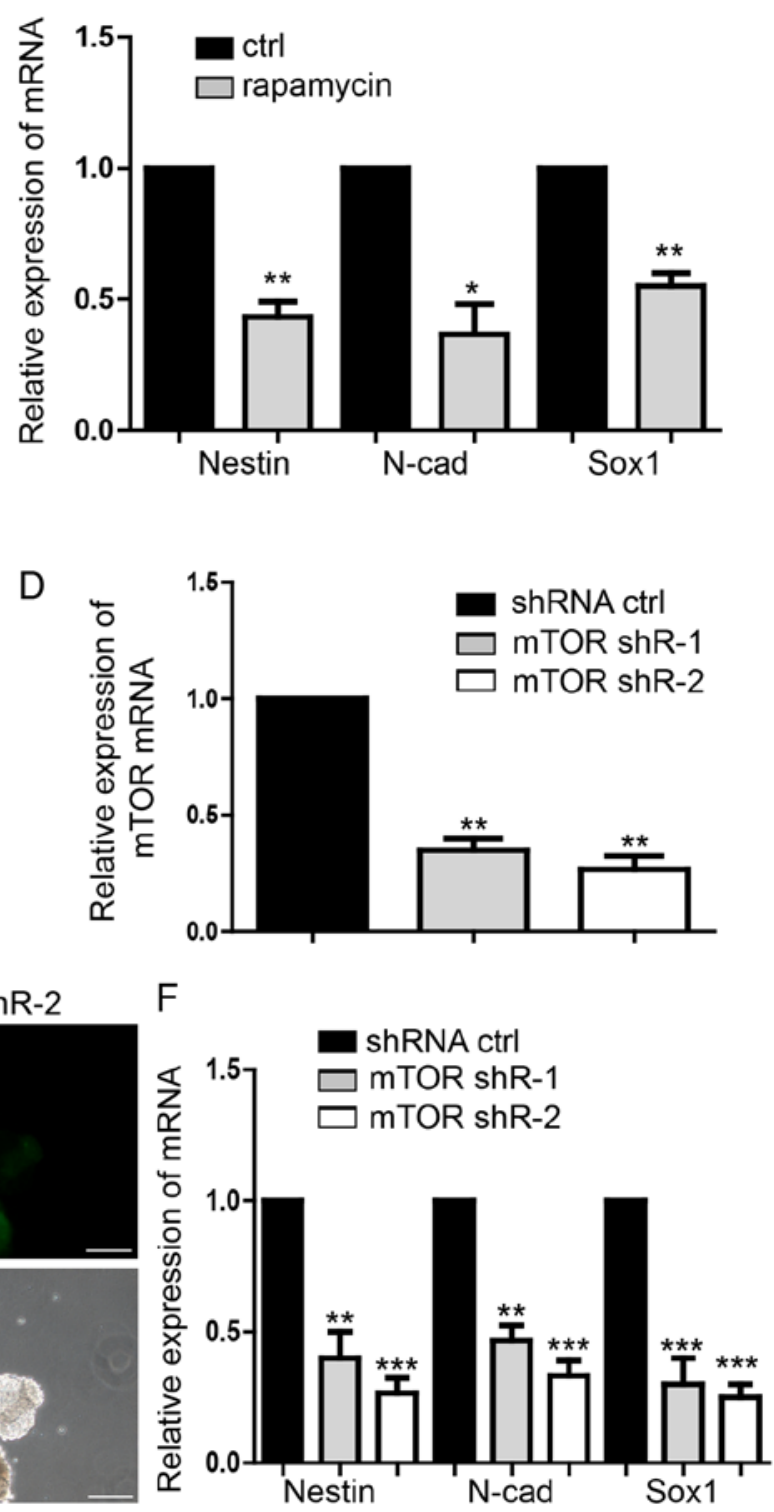

Figure 3. Inhibition of mTOR suppresses neural differentiation. (A) Representative images of neural differentiation in the rapamycin-treatment and control groups. (B) Expression of NPCs markers of Nestin, N-cad and Sox1 by RT-qPCR. (C) Flow cytometry analysis of rapamycin-treatment and control group. (D and E) Detection of the mTOR knockdown effect by shRNA, which suppressed neural differentiation. (F) Expression levels of NPCs markers, as measured by RT-qPCR. (G) Flow cytometry analysis indicating less NPCs following transfection with shRNA. Scale bar, $100 \mu \mathrm{m}$. Data are presented as the mean \pm standard deviation $(\mathrm{n}=4) .{ }^{*} \mathrm{P}<0.05,{ }^{* *} \mathrm{P}<0.01$ and ${ }^{* * * *} \mathrm{P}<0.001$ vs. the ctrl. Ctrl, control; N-cad, N-cadherin; NPC, neural progenitor cell; m-TOR, mammalian target of rapamycin; p, phosphorylated; RT-qPCR, reverse transcription-quantitative polymerase chain reaction; shR, short hairpin RNA; Sox, sex-determining region Y-box.

Ketamine activates the mTOR signaling pathway. In order to detect the downstream targets of ketamine, western blotting was performed, which demonstrated the significant upregulation of p-mTOR (Fig. 2A) and of its downstream target,
p-70S6K compared with the control group (Fig. 2B), without influencing their total expression levels. Inhibition of the NMDA signaling pathway by the NMDA receptor antagonist MK-801 significantly decreased p-mTOR expression levels 
A
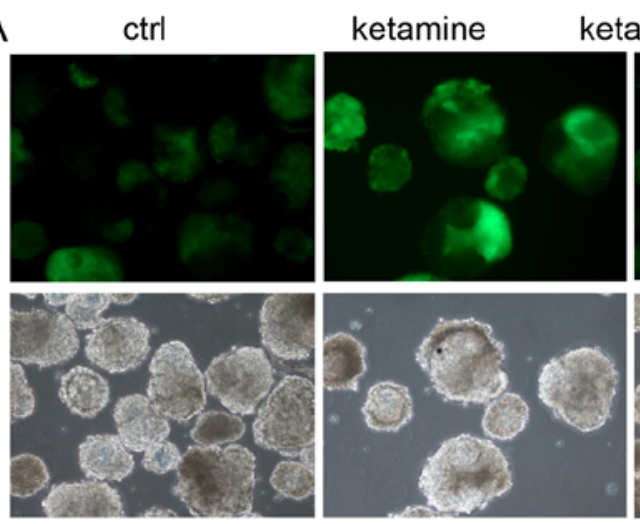

\section{C}

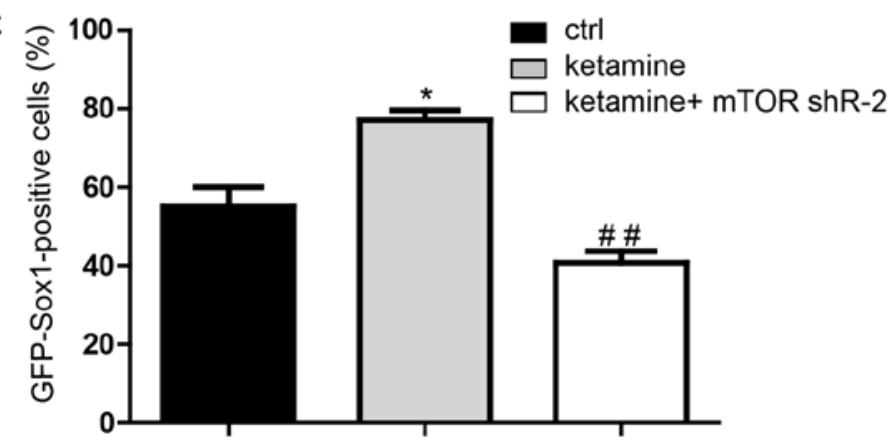

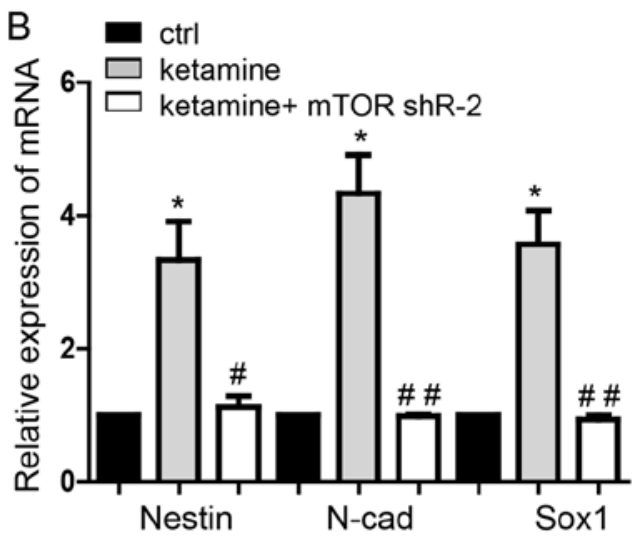

Figure 4. mTOR mediates the function of ketamine-regulated neural differentiation. (A) Representative images of neural differentiation in rescue experiments. (B) Downregulation of mTOR restored neural progenitor cell marker expression levels, in the ketamine-treatment group. (C) Flow cytometry assay also indicated that mTOR knockdown inhibited the neural differentiation promoted by ketamine. Scale bar, $100 \mu \mathrm{m}$. Data are presented as the mean \pm standard deviation ( $\mathrm{n}=3)$. ${ }^{*} \mathrm{P}<0.05$ vs. respective ctrl; ${ }^{\#} \mathrm{P}<0.05,{ }^{\#} \mathrm{P}<0.01$ vs. respective ketamine. Ctrl, control, cells transfected with the empty vector pLKO.1 and treated with $0.9 \%$ saline; mTOR, mammalian target of rapamycin; shR, short hairpin RNA; Sox, sex-determining region Y-box.

(Fig. 2C), which suggested that inhibition of NDMA signaling is not able to increase the mTOR expression level. Rapamycin $(50 \mu \mathrm{M})$ was used to notably reduce the activation of mTOR signaling caused by ketamine. Subsequently, the cells were treated with ketamine and rapamycin together to perform the rescue experiments and it was identified that rapamycin was able to block the p-mTOR expression level increased by ketamine (Fig. 2D).

Inhibition of the mTOR suppresses neural differentiation. The neural differentiation of mESCs was analyzed and rapamycin $(50 \mu \mathrm{M})$ was added to the medium to investigate whether the number of NSCs was decreased compared with the control group on day 7 (Fig. 3A). Expression levels of Nestin, Sox1, N-cad were significantly downregulated by rapamycin compared with the control (Fig. 3B). Flow cytometry assay also indicated significantly fewer GFP-Sox1-positive cells following rapamycin treatment compared with the control (Fig. 3C). Transfection with mTOR-shRNA during the differentiation of mESCs to NSCs (Fig. 3D) notably suppressed neural differentiation (Fig. 3E). The expression levels of the NSCs markers were significantly downregulated in response to mTOR silencing compared with in the control (Fig. 3F). Finally, the proportion of GFP-SOX1-positive cells in the mTOR knockdown groups were significantly decreased compared with the control, as measured by flow cytometry (Fig. 3G). These results suggested that inhibition of mTOR signaling was able to significantly repress neural differentiation, which is contrary to the function of ketamine and suggested the possible regulatory mechanism of ketamine/mTOR signaling during neural differentiation.

mTOR mediates the function of ketamine-regulated neural differentiation. Transfection with mTOR-shRNA (Fig. 3D) demonstrated that shRNA-2 induced more of a decrease of average mTOR expression and a more marked inhibitory effect on neural differentiation compared with shRNA-1. Therefore, shRNA-2 was selected for further study. Downregulation of mTOR significantly inhibited the promotion of neural differentiation induced by ketamine, on day 7 (Fig. 4A). Expression levels of NSCs markers were significantly restored by mTOR knockdown in the ketamine-treatment group (Fig. 4B). Flow cytometry also confirmed the rescue effect of mTOR downregulation following ketamine treatment (Fig. 4C). These results suggested that repression of mTOR blocked neural differentiation promoted by ketamine, which suggested the novel involvement of the ketamine/mTOR signaling pathway during neural differentiation.

\section{Discussion}

In the present study, it was revealed that ketamine activated mTOR to promote the neural differentiation of mESCs, providing the theoretical basis for the rational use of ketamine.

Ketamine, a widely used anesthetic, has potential neurodegenerative and long-term cognitive deficits, affecting brain development (7,36-39). Methods of safe ketamine 
application is an important research goal in clinical practice. The effects of ketamine are not only dependent on its dose, but also on the frequency of exposure (40-43). Ketamine has a relative neuroprotective function by relieving pain and inhibiting inflammation (44). Ketamine serves an important role in regulating nerve development (16). In the present study, ketamine at $50 \mathrm{nM}$ promoted neural differentiation and upregulated NSC marker expression levels. The process of neural differentiation occurs during early development $(45,46)$. The present results additionally demonstrated the positive effects of ketamine at a low dose, suggesting the safe clinical use in surgery for pregnant patients and children in the future.

ESCs have been extensively used for studying development, particularly neural development (47-51). Numerous genes serve an important role in the differentiation into neural stem cells $(52,53)$. A recent study demonstrated that fibronectin type III domain-containing 5 facilitated neural differentiation by increasing the expression of brain derived neurotrophic factor (54). Zfhxlb gene expression has been confirmed to be notably upregulated via the fibroblast growth factor signaling pathway in mESCs cultured in a permissive neural-inducing environment (19). Ketamine was proposed to regulate mTOR activity by upregulating the expression levels of p-mTOR in the present study. This was reversed by adding the mTOR inhibitor rapamycin or by downregulating mTOR. This suggested a potential molecular mechanism of ketamine regulation; however, further investigation is required.

In neural progenitors, insulin has been demonstrated to induce neurogenesis of NPCs by activating mTOR (26). mTOR is also needed for the of dendritic arbors development and stabilization in the newly born olfactory bulb neurons (55). The mTOR signaling pathway was reported to mediate valproic acid-induced neural differentiation of NSCs (56). Inhibition of the mTOR signaling pathway by rapamycin was observed to suppress neural differentiation in the present study. The promotion of neural differentiation caused by ketamine was also inhibited by silencing mTOR. The expression of Nestin, Sox1 and N-cad was also restored by downregulating mTOR. The NMDA signaling pathway, was inhibited during the neural differentiation and the levels of p-mTOR were also suppressed. This result indicated the regulatory function of ketamine via a non-NMDA signaling pathway during neural differentiation. However, a limitation of the present study is that whether the NMDA receptor may influence neural differentiation remains unknown. mTOR complex 1 (mTORC1) was closely associated with the neuron-associated biological process downstream target (57). The activity of p70S6K, the downstream target of mTORC1, was increased by ketamine, indicating that it may participate in the regulation of ketamine. These results determined that the ketamine/mTOR signaling pathway regulated the neural differentiation process of NSCs derived from mESCs; however, further investigation is required.

In summary, the present study revealed the ketamine/ mTOR signaling pathway on regulating the neural differentiation and suggested a potential dose of ketamine. The ketamine/ mTOR signaling pathway needs to be further investigated for its potential use in clinic.

\section{Acknowledgements}

The authors would like to thank Dr Xiaoqing Zhang (Tongji University, Shanghai, China) for providing the Sox1-promoter-GFP 46C mESCs.

\section{Funding}

The present study was supported by the National Natural Science Foundation of China (grant no. 81571028). Research funds were from the Shanghai Municipal Science and Technology Commission (grant no. 16XD1401800) and the Natural Science Foundation of Shanghai (grant nos. 17ZR1416400 and 17DZ1205403).

\section{Availability of data and materials}

The datasets used and/or analyzed during the current study are available from the corresponding author on reasonable request.

\section{Authors' contributions}

$\mathrm{XZ}$ performed the experiments. XL performed the reverse transcription-quantitative polymerase chain reaction assays and wrote parts of the manuscript. LZ performed the western blotting. JY conducted the statistical analysis. RH performed microscopy. YS cultured and prepared the cells. SX analyzed the expression level of western blot. HJ provided guidance and analyzed some of the data. All authors read and approved the final manuscript.

\section{Ethics approval and consent to participate}

Not applicable.

\section{Patient consent for publication}

Not applicable.

\section{Competing interests}

The authors declare that they have no competing interests.

\section{References}

1. Sinner B and Graf BM: Ketamine. Handb Exp Pharmacol: 313-333, 2008.

2. Rofael HZ, Turkall RM and Abdel-Rahman MS: Immunomodulation by cocaine and ketamine in postnatal rats. Toxicology 188: 101-114, 2003.

3. Reitman E and Flood P: Anaesthetic considerations for non-obstetric surgery during pregnancy. Br J Anaesth 107 (Suppl 1): i72-i78, 2011.

4. Wilder RT, Flick RP, Sprung J, Katusic SK, Barbaresi WJ, Mickelson C, Gleich SJ, Schroeder DR, Weaver AL and Warner DO: Early exposure to anesthesia and learning disabilities in a population-based birth cohort. Anesthesiology 110: 796-804, 2009.

5. Huang H, Liu CM, Sun J, Hao T, Xu CM, Wang D and Wu YQ: Ketamine affects the neurogenesis of the hippocampal dentate gyrus in 7-day-old rats. Neurotox Res 30: 185-198, 2016.

6. Wang J, Zhou M, Wang X, Yang X, Wang M, Zhang C, Zhou S and Tang N: Impact of ketamine on learning and memory function, neuronal apoptosis and its potential association with mir-214 and pten in adolescent rats. PLoS One 9: e99855, 2014. 
7. Yan J, Huang Y, Lu Y, Chen J and Jiang H: Repeated administration of ketamine can induce hippocampal neurodegeneration and long-term cognitive impairment via the ROS/HIF-1 $\alpha$ pathway in developing rats. Cell Physiol Biochem 33: 1715-1732, 2014.

8. Hudetz JA, Patterson KM, Iqbal Z, Gandhi SD, Byrne AJ, Hudetz AG, Warltier DC and Pagel PS: Ketamine attenuates delirium after cardiac surgery with cardiopulmonary bypass. J Cardiothorac Vasc Anesth 23: 651-657, 2009.

9. Hovaguimian F, Tschopp C, Beck-Schimmer B and Puhan M: Intraoperative ketamine administration to prevent delirium or postoperative cognitive dysfunction: A systematic review and meta-analysis. Acta Anaesthesiol Scand 62: 1182-1193, 2018.

10. Nikiforuk A and Popik P: Ketamine prevents stress-induced cognitive inflexibility in rats. Psychoneuroendocrinology 40: 119-122, 2014

11. Wang CQ, Ye Y, Chen F, Han WC, Sun JM, Lu X, Guo R, Cao K, Zheng MJ and Liao LC: Posttraumatic administration of a sub-anesthetic dose of ketamine exerts neuroprotection via attenuating inflammation and autophagy. Neuroscience 343 . 30-38, 2017.

12. Hertle DN, Dreier JP, Woitzik J, Hartings JA, Bullock R, Okonkwo DO, Shutter LA, Vidgeon S, Strong AJ, Kowoll C, et al: Effect of analgesics and sedatives on the occurrence of spreading depolarizations accompanying acute brain injury. Brain 135: 2390-2398, 2012.

13. Koerner IP and Brambrink AM: Brain protection by anesthetic agents. Curr Opin Anaesthesiol 19: 481-486, 2006.

14. Sanders RD, Ma D, Brooks $P$ and Maze M: Balancing paediatric anaesthesia: Preclinical insights into analgesia, hypnosis, neuroprotection, and neurotoxicity. Br J Anaesth 101: 597-609, 2008.

15. Murrough JW: Ketamine for depression: An update. Biol Psychiatry 80: 416-418, 2016.

16. Dong C, Rovnaghi CR and Anand KJ: Ketamine alters the neurogenesis of rat cortical neural stem progenitor cells. Crit Care Med 40: 2407-2416, 2012.

17. Bosnjak ZJ, Yan Y, Canfield S, Muravyeva MY, Kikuchi C, Wells CW, Corbett JA and Bai X: Ketamine induces toxicity in human neurons differentiated from embryonic stem cells via mitochondrial apoptosis pathway. Curr Drug Saf 7: 106-119, 2012.

18. Czechanski A, Byers C, Greenstein I, Schrode N, Donahue LR, Hadjantonakis AK and Reinholdt LG: Derivation and characterization of mouse embryonic stem cells from permissive and nonpermissive strains. Nat Protoc 9: 559-574, 2014.

19. Dang LT, Wong L and Tropepe V: Zfhxlb induces a definitive neural stem cell fate in mouse embryonic stem cells. Stem Cells Dev 21: 2838-2851, 2012

20. Tang S, Huang G, Fan W, Chen Y, Ward JM, Xu X, Xu Q, Kang A, McBurney MW, Fargo DC, et al: SIRT1-mediated deacetylation of CRABPII regulates cellular retinoic acid signaling and modulates embryonic stem cell differentiation. Mol Cell 55: 843-855, 2014.

21. Zhang L, Zheng Y, Sun Y, Zhang Y, Yan J, Chen Z and Jiang H: MiR-134-Mbd3 axis regulates the induction of pluripotency. J Cell Mol Med 20: 1150-1158, 2016.

22. Meng SS, Guo FM, Zhang XW, Chang W, Peng F, Qiu HB and Yang Y: mTOR/STAT-3 pathway mediates mesenchymal stem cell-secreted hepatocyte growth factor protective effects against lipopolysaccharide-induced vascular endothelial barrier dysfunction and apoptosis. J Cell Biochem 120: 3637-3650, 2018.

23. Nguyen K, Yan Y, Yuan B, Dasgupta A, Sun JC, Mu H, Do KA, Ueno NT, Andreeff $M$ and Battula VL: ST8SIA1 regulates tumor growth and metastasis in TNBC by activating the FAK-AKT-mTOR signaling pathway. Mol Cancer Ther 17: 2689-2701, 2018

24. Wang Y, Ma J, Qiu W, Zhang J, Feng S, Zhou X, Wang X, Jin L, Long K, Liu L, et al: Guanidinoacetic acid regulates myogenic differentiation and muscle growth through miR-133a-3p and miR-1a-3p Co-mediated Akt/mTOR/S6K signaling pathway. Int J Mol Sci 19: pii: E2837, 2018.

25. Magri L and Galli R: Mtor signaling in neural stem cells: From basic biology to disease. Cell Mol Life Sci 70: 2887-2898, 2013.

26. Han J, Wang B, Xiao Z, Gao Y, Zhao Y, Zhang J, Chen B, Wang X and Dai J: Mammalian target of rapamycin (mTOR) is involved in the neuronal differentiation of neural progenitors induced by insulin. Mol Cell Neurosci 39: 118-124, 2008.

27. Dai J, Bercury KK and Macklin WB: Interaction of mTOR and Erk1/2 signaling to regulate oligodendrocyte differentiation. Glia 62: 2096-2109, 2014
28. Ka M, Smith AL and Kim WY: MTOR controls genesis and autophagy of GABAergic interneurons during brain development. Autophagy 13: 1348-1363, 2017.

29. Xing $\mathrm{J}$ and $\mathrm{Lu} \mathrm{J}$ : Effects of mTOR on neurological deficits after transient global ischemia. Transl Neurosci 8: 21-26, 2017.

30. Barraud P, Thompson L, Kirik D, Bjorklund A and Parmar M: Isolation and characterization of neural precursor cells from the Sox1-GFP reporter mouse. Eur J Neurosci 22: 1555-1569, 2005.

31. Reinés A, Bernier LP, McAdam R, Belkaid W, Shan W, Koch AW, Séguéla P, Colman DR and Dhaunchak AS: N-cadherin prodomain processing regulates synaptogenesis. J Neurosci 32 : 6323-6334, 2012.

32. Zhang J and Jiao J: Molecular biomarkers for embryonic and adult neural stem cell and neurogenesis. Biomed Res Int 2015: $727542,2015$.

33. Ying QL, Stavridis M, Griffiths D, Li M and Smith A: Conversion of embryonic stem cells into neuroectodermal precursors in adherent monoculture. Nat Biotechnol 21: 183-186, 2003.

34. Watanabe K, Kamiya D, Nishiyama A, Katayama T, Nozaki S, Kawasaki H, Watanabe Y, Mizuseki K and Sasai Y: Directed differentiation of telencephalic precursors from embryonic stem cells. Nat Neurosci 8: 288-296, 2005.

35. Livak KJ and Schmittgen TD: Analysis of relative gene expression data using real-time quantitative pcr and the 2(-delta delta $\mathrm{c}(\mathrm{t}))$ method. Methods 25: 402-408, 2001.

36. Gass N, Schwarz AJ, Sartorius A, Schenker E, Risterucci C, Spedding M, Zheng L, Meyer-Lindenberg A and Weber-Fahr W: Sub-anesthetic ketamine modulates intrinsic BOLD connectivity within the hippocampal-prefrontal circuit in the rat. Neuropsychopharmacology 39: 895-906, 2014

37. Yan J and Jiang H: Dual effects of ketamine: Neurotoxicity versus neuroprotection in anesthesia for the developing brain J Neurosurg Anesthesiol 26: 155-160, 2014.

38. Theurillat R, Larenza MP, Feige K, Bettschart-Wolfensberger R and Thormann W: Development of a method for analysis of ketamine and norketamine enantiomers in equine brain and cerebrospinal fluid by capillary electrophoresis. Electrophoresis 35 : 2863-2869, 2014

39. Li J, Wang B, Wu H, Yu Y, Xue G and Hou Y: 17ß-estradiol attenuates ketamine-induced neuroapoptosis and persistent cognitive deficits in the developing brain. Brain Res 1593: 30-39, 2014.

40. Permoda-Osip A, Kisielewski J, Bartkowska-Sniatkowska A and Rybakowski JK: Single ketamine infusion and neurocognitive performance in bipolar depression. Pharmacopsychiatry 48: 78-79, 2015

41. Diamond PR, Farmery AD, Atkinson S, Haldar J, Williams N, Cowen PJ, Geddes JR and McShane R: Ketamine infusions for treatment resistant depression: A series of 28 patients treated weekly or twice weekly in an ECT clinic. J Psychopharmacol 28: 536-544, 2014

42. Neri CM, Pestieau SR and Darbari DS: Low-dose ketamine as a potential adjuvant therapy for painful vaso-occlusive crises in sickle cell disease. Paediatr Anaesth 23: 684-689, 2013.

43. Shibuta S, Morita T, Kosaka J, Kamibayashi T and Fujino Y: Only extra-high dose of ketamine affects l-glutamate-induced intracellular $\mathrm{Ca}(2+)$ elevation and neurotoxicity. Neurosci Res 98: 9-16, 2015 .

44. Bhutta AT, Schmitz ML, Swearingen C, James LP, Wardbegnoche WL, Lindquist DM, Glasier CM, Tuzcu V, Prodhan P, Dyamenahalli U, et al: Ketamine as a neuroprotective and anti-inflammatory agent in children undergoing surgery on cardiopulmonary bypass: A pilot randomized, double-blind, placebo-controlled trial. Pediatr Crit Care Med 13: 328-337, 2012.

45. Sheridan MA and McLaughlin KA: Dimensions of early experience and neural development: Deprivation and threat. Trends Cogn Sci 18: 580-585, 2014.

46. Kolb B, Mychasiuk R and Gibb R: Brain development, experience, and behavior. Pediatr Blood Cancer 61: 1720-1723, 2014.

47. Lu AQ, Popova EY and Barnstable CJ: Activin signals through SMAD2/3 to increase photoreceptor precursor yield during embryonic stem cell differentiation. Stem Cell Reports 9: 838-852, 2017.

48. Liu Q, Wang G, Chen Y, Li G, Yang D and Kang J: A miR-590/ Acvr2a/Rad51b axis regulates DNA damage repair during mESC proliferation. Stem Cell Reports 3: 1103-1117, 2014.

49. Xu N, Papagiannakopoulos T, Pan G, Thomson JA and Kosik KS MicroRNA-145 regulates OCT4, SOX2, and KLF4 and represses pluripotency in human embryonic stem cells. Cell 137: 647-658, 2009. 
50. Lei J, Yuan Y,Lyu Z, Wang M,Liu Q, Wang H, Yuan L and Chen H: Deciphering the role of sulfonated unit in heparin-mimicking polymer to promote neural differentiation of embryonic stem cells. ACS Appl Mater Interfaces 9: 28209-28221, 2017.

51. Gazina EV, Morrisroe E, Mendis GDC, Michalska AE, Chen J, Nefzger CM, Rollo BN, Reid CA, Pera MF and Petrou S: Method of derivation and differentiation of mouse embryonic stem cells generating synchronous neuronal networks. J Neurosci Methods 293: 53-58, 2018.

52. Fischer U, Keller A, Voss M, Backes C, Welter C and Meese E: Genome-wide gene amplification during differentiation of neural progenitor cells in vitro. PLoS One 7: e37422, 2012.

53. Mateo JL, van den Berg DL, Haeussler M, Drechsel D, Gaber ZB, Castro DS, Robson P, Lu QR, Crawford GE, Flicek P, et al: Characterization of the neural stem cell gene regulatory network identifies OLIG2 as a multifunctional regulator of self-renewal. Genome Res 25: 41-56, 2015.

54. Forouzanfar M, Rabiee F, Ghaedi K, Beheshti S, Tanhaei S, Shoaraye Nejati A, Jodeiri Farshbaf M, Baharvand H and Nasr-Esfahani MH: Fndc5 overexpression facilitated neural differentiation of mouse embryonic stem cells. Cell Biol Int 39: 629-637, 2015.
55. Skalecka A, Liszewska E, Bilinski R, Gkogkas C, Khoutorsky A, Malik AR, Sonenberg N and Jaworski J: mTOR kinase is needed for the development and stabilization of dendritic arbors in newly born olfactory bulb neurons. Dev Neurobiol 76: 1308-1327, 2016. 56. Zhang X, He X, Li Q, Kong X, Ou Z, Zhang L, Gong Z, Long D, Li J, Zhang M, et al: PI3K/AKT/mTOR signaling mediates valproic acid-induced neuronal differentiation of neural stem cells through epigenetic modifications. Stem Cell Reports 8: 1256-1269, 2017

57. Polchi A, Magini A, Meo DD, Tancini B and Emiliani C: mTOR signaling and neural stem cells: The tuberous sclerosis complex model. Int J Mol Sci 19: pii: E1474, 2018.

(i) $($ This work is licensed under a Creative Common Attribution-NonCommercial-NoDerivatives 4.0 International (CC BY-NC-ND 4.0) License. 